\title{
The sun shadow positioning
}

\author{
Limengchen \\ School of The North China Electric Power University,Baoding 071000,China \\ 1491239082@qq.com
}

Keywards: The sun's shadow,The geographical location,Genetic algorithm.

\begin{abstract}
In view of the problem, To study the curve of the solar shadow over time, the first step is to set each parameter, establishing a mathematical model of the change in length of the shadow, according to the longitude and the time difference work out the real solar time and the solar hour angle are, according to the date and reach the solar declination angle, combining the latitude, solar declination angle、 the solar hour angle to calculate the solar zenith angle, then the length of the sun shadow is obtained by the known rod length, and the change curve of the shadow length with time is drawn by MATLAB.
\end{abstract}

\section{Introduction}

Shadow length changes of the mathematical model of the shadow length about the change rule of each parameter, and establish the model of application you draw on October 22, 2015, Beijing time between 9:00-15:00 Tian'anmen Square (54 minutes and 26 seconds, 39 degrees north latitude 23 minutes 29 seconds 116 degrees east longitude) length of 3 meters tall straight rod of the sun's shadow change curve.

\section{Models for the length of the shadow}

\subsection{Symbols,Definitions \&Assumptions}

- Symbols\&Definitions

\begin{tabular}{|c|c|}
\hline Symbols & Definitions \\
\hline$H$ & The length of the rods \\
\hline l & $\begin{array}{c}\text { The length of the straight rod sun } \\
\text { shadow }\end{array}$ \\
\hline$\alpha$ & Solar hour angle \\
\hline$\delta$ & Solar Declination Angle \\
\hline$h$ & Solar zenith angle \\
\hline$S T$ & True solar time \\
\hline$T$ & Beijing time at a certain time \\
\hline$\varepsilon$ & Longitude \\
\hline$\phi$ & Latitude \\
\hline Et & Time difference $(\mathrm{min})$ \\
\hline$\theta$ & Day Angle \\
\hline$N$ & Ahargana \\
\hline$t$ & $t=N-N_{0}$ \\
\hline Year & Year \\
\hline$\lambda$ & Solar azimuth \\
\hline
\end{tabular}


- Assumptions

(1) The earth is a regular sphere;

(2) We suppose the sun's rays is completely parallel;

(3) We assume that the ground of the shadow is completely level;

(4) We assume the sun declination angle is constant throughout the day;

(5) Common year is 365 days, leap year is 366 days;

\subsection{Model construction for the shadow length change}

(1) Calculating the solar zenith angle

Solar zenith angle refers to the incident direction of the sun and the angle between the ground plane, Professionally speaking, the solar zenith angle is the angle between the sun and the earth's surface, which is connected with the inner earth through the earth's surface.

According to the analysis, in a certain place A, at local accurate time noon twelve o 'clock, the sun shone directly on the place,assuming that the earth is a regular sphere.

Make a brief as follows:

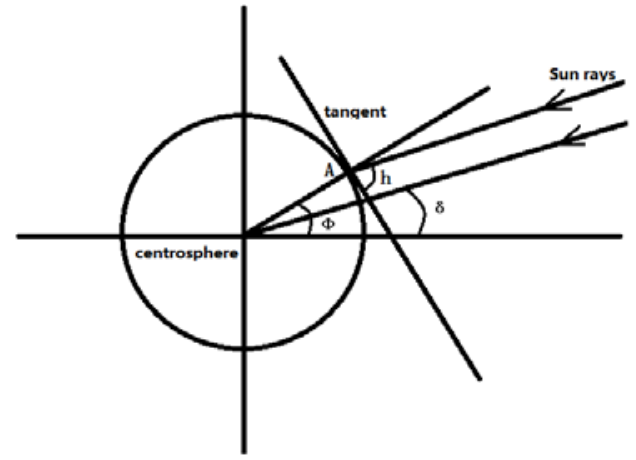

Figure 1 Diagram of a point A in earth's surface at noon

Using the geometric theorem can easily infer $h=90^{\circ}-|\phi-\delta|$ that is when the sun at the zenith, the solar zenith angle is the latitude minus the solar declination angle after complement.

To derive the solar zenith angle $h$ of any moment in a day as follows:

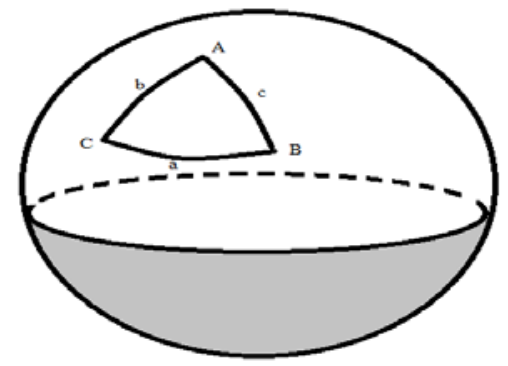

Figure 2 Calculation of the solar zenith angle at any moment

In the spherical triangle $\mathrm{ABC}$, the cosine formula for the example of $\mathrm{B}$ side is:

$$
\cos b=\cos a \cos c+\sin a \sin c \cos B
$$

The other sides of the corner with the corresponding substitution.Thus infer formula the solar zenith angle at any time:

$$
\cos \left(90^{\circ}-h\right)=\cos \left(90^{\circ}-\delta\right) \cos \left(90^{\circ}-\phi\right)+\sin \left(90^{\circ}-\delta\right) \sin \left(90^{\circ}-\phi\right) \cos \alpha
$$

The reduction is as follows:

$$
\sinh =\sin \delta \sin \phi+\cos \delta \cos \phi \cos \alpha
$$

In the formula, $h$ represents the solar zenith angle, $\alpha$ indicates the solar hour angle, $\delta$ indicates solar declination angle , and $\phi$ represents the local geographic latitude.

Special case: at noon at local time, $\alpha=0^{\circ}$, in formula(3), the value of $\cos \alpha$ is 1 , the calculating formula of Angle of the sun at this time as follows:

$$
\sinh =\sin \delta \sin \phi+\cos \delta \cos \phi
$$


(2) Calculating the solar hour angle

$$
h=90^{\circ}-|\phi-\delta|
$$

Solar hour angle means the hour angel of surface center,namely the angular distance from the point of observation celestial meridian along the celestial equator to the sun.

Calculation formula is:

$$
\begin{gathered}
\alpha=15 \times(S T-12) \\
S T=T+\frac{\left(\varepsilon-120^{\circ}\right)}{15^{\circ}}+E t \\
E t=0.0028-1.9857 \sin \theta+9.9059 \sin 2 \theta-7.0924 \cos \theta-0.6882 \cos 2 \theta \\
\theta=2 \pi t / 365.2422 \\
t=N-N_{0} \\
N_{0}=79.6764+0.2422 \times(\text { Year-1985) INT }[(\text { Year-1985) } / 4]
\end{gathered}
$$

(3) Calculating the solar declination angle

$$
\begin{aligned}
& E d=0.3723+23.2567 \sin \theta+0.1149 \sin 2 \theta-0.1712 \sin 3 \theta-0.758 \cos \theta \\
& +0.3656 \cos 2 \theta+0.0201 \cos 3 \theta
\end{aligned}
$$

(4) Calculating the solar azimuth

The solar azimuth is the angle between the projection and the south, which is expressed by $\lambda$,the calculation formula is as follows:

$$
\cos \lambda=\frac{\cos \delta \sin \alpha}{\cosh }
$$

(5) Description of the change curve of the solar shadow length of the straight bar Simple changes can be inferred straight sun shadow is a curve, draw the day the sun shadow change chart bar are as follows:

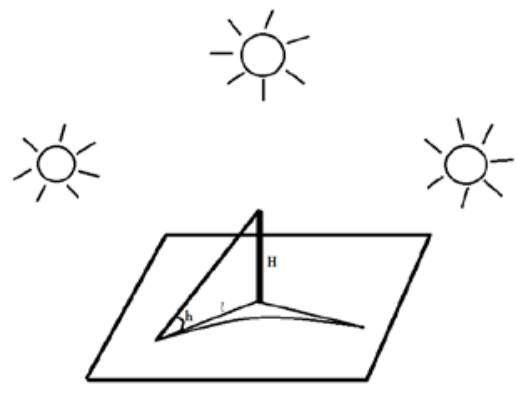

Figure 3 The variation of the sun's shadow over the course of a day.

According to the geometric theorem, The length of the straight rod sun shadow $l$ can be calculated:

$$
l=\frac{H}{\tanh }
$$

Combined with the above formula can come to the sun's shadow on the parameters of the function.

\subsection{Result and analysis}

By using the software of MATLAB with the exist data ,the function of the length of the sun shadow of a straight rod as a function of time is as follows:

$$
l=3 \frac{(-0.0643+0.9232 \times \cos (-0.78+(t-9) \times \pi / 12))}{\sqrt{1-(-0.0643+0.9232 \times \cos (-0.78+(t-9) \times \pi / 12))}}
$$




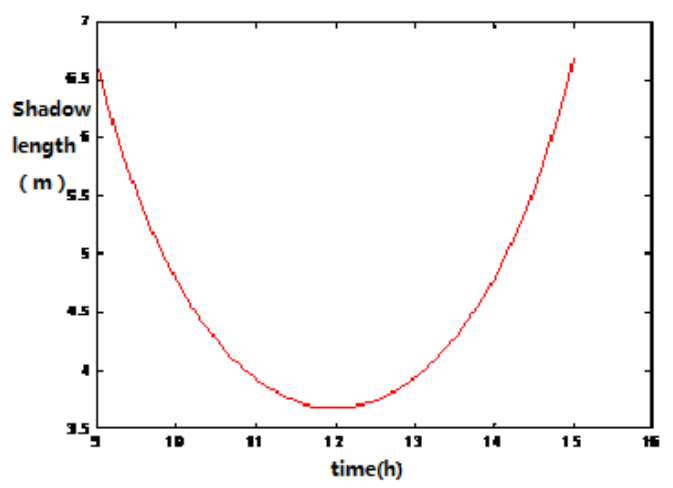

Figure 4 Straight rod length of the sun shadow along with the change of time curve At the low end of the straight rod as the origin, to the south for the $\mathrm{X}$ axis, the East is the $\mathrm{Y}$ axis, The trajectory of the shadow vertex is obtained:

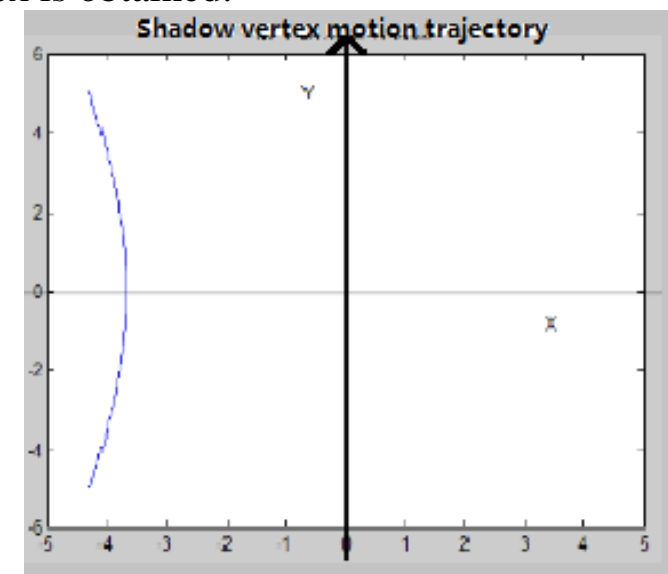

Figure 5 The sun's shadow vertex of the trajectory

\section{Reference}

[1] Liu Xuefu, basic astronomy, higher education press, 2004.7

[2] Wang Shuiping genetic algorithm theory, application and software, Xi'an Jiao Tong University, 2002.1

[3] Zhuo Jinwu, Li Biwen, application of MATLAB in mathematical modeling ,second ed., Beihang University press, 2014.9

[4] Jiang Qiyuan, mathematical model (Fourth Edition), higher education press, 2011.7.28 